\title{
Design of delay-based output-feedback controllers optimizing a quadratic cost function via the delay Lyapunov matrix *
}

\author{
Marco A. Gomez ${ }^{a}$, Wim Michiels ${ }^{a}$, Sabine Mondié ${ }^{b}$ \\ ${ }^{a}$ Department of Computer Science, KU Leuven, Leuven, 3001 Belgium \\ ${ }^{\mathrm{b}}$ Department of Automatic Control, CINVESTAV-IPN, Mexico City, 07360 Mexico
}

\begin{abstract}
A novel approach for the design of delayed output-feedback controllers that optimize a quadratic cost function is presented. The proposal is based on the formulation of the optimization of the quadratic performance criterion as a minimization problem whose objective function is given in terms of the so-called delay Lyapunov matrix. The computation of the delay Lyapunov matrix sensitivity with respect to the controller gains allows obtaining the gradient of the objective function and solving the minimization problem within a gradient-based optimization framework. The potential of the approach is shown by some examples of practical relevance.
\end{abstract}

Key words: Quadratic performance index; delayed output-feedback; delay Lyapunov matrix.

\section{Introduction}

It is well known that delays appear in a number of physical phenomena and engineering applications, and in general they are not considered to be desirable. However, they are not always detrimental. In the last decades a number of research works have demonstrated that the deliberated introduction of delays can be useful for stabilization. See, for instance, [34], [26], [27], where Proportional-Retarded controllers are proposed, or [23] and [17], where it is shown that static output-feedback with delays can stabilize a chain of integrators or oscillators. See also [11] and [32].

As in the delay-free controllers case, the study of the LQR (Linear Quadratic Regulator) problem is of prime interest and a difficult task. Since the contributions in [18] and [29] over 50 years ago, it is known that the solution to the LQR problem for systems with delays is given in terms of a complex system of partial differential equations, and that the optimal control law is expressed in terms of distributed delays. Guaranteed cost controls with simpler structures considering delays in the input and output have been proposed in past years (see, for

* This paper was not presented at any IFAC meeting. Corresponding author Marco A. Gomez. Tel. +3216323164.

Email addresses: marco.gomez@cs.kuleuven. be (Marco A. Gomez), Wim.Michiels@cs.kuleuven.be (Wim Michiels), smondie@ctrl.cinvestav.mx (Sabine Mondié). instance, [24], [10], [6], and the references therein). The recent monograph by [19] gives a consistent overview of this kind of controllers for different classes of delay systems. A common feature of guaranteed cost controls available in the literature is that they are obtained as solution of Riccati type equations or by using LMI (Linear Matrix Inequality) techniques. In this approach, the structure of the controller is proposed and the gains are sought to satisfy a given performance level.

In this paper, we address the design problem of delayed output-feedback controllers optimizing a quadratic performance criterion from a novel viewpoint by using the delay Lyapunov matrix. The so-called delay Lyapunov matrix, which plays the role of the Lyapunov matrix in the delay-free case and is solution of three equations known as dynamic, symmetry and algebraic properties [16], has proven to be a powerful tool for the study of the stability [9], [7], [13], robustness [1], and $\mathcal{H}_{2}$ norm [15], [14], of time-delay systems. It has also been used for computing guaranteed cost controls for systems with delay in the state, but neither in the input nor the output in [31] and [30].

The merit of the new approach lies in the restatement of the design problem as a minimization problem whose objective function is given in terms of a sum of delay Lyapunov matrices. If the initial state of the system is known, it is shown that the minimization problem is smooth with respect to the controller parameters and can be solved by using standard gradient-based opti- 
mization techniques. However, a more realistic scenario is when one has no knowledge on the initial conditions, for which we consider the worst-case delayed output feedback with respect to unknown initial conditions. In this case, the objective function is determined by the largest eigenvalue of a symmetric matrix, and the minimization problem may be non-smooth. In order to solve this problem, we make use of gradient-based optimization techniques for non-smooth non-convex functions introduced in [20] (see [4] and [21] for some successful applications of these techniques). The required gradient wherever the objective function is differentiable is computed from the sensitivity of the delay Lyapunov matrix with respect to the parameters of the controller, which is obtained as a solution of an ordinary differential equation system with boundary conditions. This result extends the one delay case presented in [14] to the multiple commensurate delays case.

Unlike the previously mentioned LMI approach, no upper bound on the performance index is set and sufficient conditions for meeting this bound are derived, but the performance index is directly minimized as a function of the parameters of the controller. Furthermore, given a starting controller such that the closedloop system is exponentially stable, the algorithm converges to the optimal controller within the class of delayed feedback-output controllers in a neighborhood of the starting point, and to a local minimum of the performance index.

The remainder of the paper is organized as follows. In the next section, we formally state the problem. Section 3 is devoted to the introduction of basic definitions and concepts concerning the delay Lyapunov matrix framework. The main result of the paper is established in Section 4 . There, the performance indexes to be minimized and their gradients are characterized by the delay Lyapunov matrix, and the employed numerical optimization techniques are explained. In Section 5, we illustrate the effectiveness and potential of the proposed approach by some examples of practical relevance, namely, in the design of Proportional-Retarded controllers and in the stabilization of a triple integrator. We end the paper with some concluding remarks and possible future work.

Throughout the paper, the notation $\|\cdot\|$ stands for the Euclidian norm. The resultant matrix of two polynomials $P$ and $Q$ is denoted by $R^{\otimes}(P, Q)$, see [12]. The classical Kronecker product of two matrices $A$ and $B$ is represented by the symbol $\otimes$, and the vectorization of a matrix $A$ is denoted by $\operatorname{vec}(A)$. The notation $\frac{\partial P(t, \mathbf{f})}{\partial f_{i}}$ and $P^{\prime}(t, \mathbf{f})$ stand for the matrix obtained by taking the partial derivative of each element of a matrix $P$ with respect to the $i-t h$ element of vector $\mathbf{f}$ and with respect to $t$, respectively. The symbol $\nabla g$ denotes the gradient of a function $g: \mathbb{R}^{n} \mapsto \mathbb{R}$ and $\lambda_{\max }(A)$ represents the largest eigenvalue of a matrix $A$. The identity matrix of dimension $n$ and the null matrix of $m$ rows and $n$ columns are denoted by $I_{n}$ and $0_{n \times m}$, respectively.

\section{Problem formulation}

We consider system

$$
\begin{aligned}
\dot{x}(t) & =A x(t)+B u(t), t \geqslant 0, \\
y(t) & =C x(t), \\
x(0) & =x_{0},
\end{aligned}
$$

where $x(t) \in \mathbb{R}^{n}$ represents the state, $u(t) \in \mathbb{R}^{n_{b}}$ is the input of the system, $y(t) \in \mathbb{R}^{n_{c}}$ is the output, $A \in \mathbb{R}^{n \times n}$, $B \in \mathbb{R}^{n \times n_{b}}$ and $C \in \mathbb{R}^{n_{c} \times n}$, and a delay-based control of the form

$$
u(t)=\sum_{i=0}^{m} F_{i} y\left(t-\tau_{i}\right),
$$

where $0=\tau_{0}<\tau_{1}<\ldots<\tau_{m}=\tau$ are commensurate delays (i.e. there exists a number $h$ such that $\tau_{i}=i h$ ) and the matrices $F_{i} \in \mathbb{R}^{n_{b} \times n_{c}}$. Notice that the state information of system (1) is available only for $t \geqslant 0$, hence assuming that $x(t)=0$ for $t<0$, the control law can be expressed as

$$
u(t)=\left\{\begin{array}{l}
\sum_{i=0}^{l} F_{i} y\left(t-\tau_{i}\right), \quad t \in\left[\tau_{l}, \tau_{l+1}\right), l=\overline{0, m-1} \\
\sum_{i=0}^{m} F_{i} y\left(t-\tau_{i}\right), \quad t \geqslant \tau .
\end{array}\right.
$$

We are interested in finding the controller gain vector

$\mathbf{f}^{T}=\left(\operatorname{vec}\left(F_{0}\right)^{T} \ldots \operatorname{vec}\left(F_{m}\right)^{T}\right)=\left(f_{1} \ldots f_{k}\right) \in \mathbb{R}^{(m+1) n_{b} n_{c}}$

that stabilizes system (1) and minimizes the performance index

$$
J\left(\mathbf{f}, x_{0}\right)=\int_{0}^{\infty}\left(x^{T}(t) Q x(t)+u^{T}(t) R u(t)\right) d t
$$

where $Q \in \mathbb{R}^{n \times n}$ and $R \in \mathbb{R}^{n_{b} \times n_{b}}$ are positive definite matrices, and $x(t)$ and $u(t)$ satisfy (1) and (2), respectively. The closed-loop system (1)-(2) is given by

$$
\dot{x}(t)=\sum_{i=0}^{m} A_{i} x\left(t-\tau_{i}\right), t \geqslant 0,
$$

where $A_{0}=A+B F_{0} C$ and $A_{i}=B F_{i} C, i=\overline{1, m}$, and its initial condition is

$$
\varphi(\theta)= \begin{cases}x_{0}, & \theta=0, \\ 0, & \theta \in[-\tau, 0) .\end{cases}
$$

We make the dependence of the matrices $A_{i}$ on the controller gains explicit by writing them as $A_{i}(\mathbf{f}), i=\overline{0, m}$. 
The spectral abscissa of system (4) is defined as

$$
\begin{aligned}
& \alpha(\mathbf{f}):= \\
& \sup \left\{\Re(s): \operatorname{det}\left(s I-\sum_{i=0}^{m} A_{i}(\mathbf{f}) e^{-s \tau_{i}}\right)=0, s \in \mathbb{C}\right\} .
\end{aligned}
$$

Notice that the minimization of (3) depends on the initial condition of system (1). As in many engineering problems the initial condition of the system is unknown, we consider the worst case within the class of unknown initial condition satisfying $\left\|x_{0}\right\| \leqslant 1$ as well. The problem addressed in this paper can be formulated as follows:

Problem 1 Solve the minimization problems

$$
\min _{\boldsymbol{f}} J\left(\boldsymbol{f}, x_{0}\right) \text { s.t. } \alpha(\boldsymbol{f})<0
$$

for a known initial condition $x_{0} \in \mathbb{R}^{n}$, and

$$
\min _{f} J_{w c}(\boldsymbol{f}) \text { s.t. } \alpha(\boldsymbol{f})<0
$$

where

$$
J_{w c}(\boldsymbol{f})=\max _{\substack{x_{0} \\\left\|x_{0}\right\| \leqslant 1}} J\left(\boldsymbol{f}, x_{0}\right)
$$

Notice that the constraints on $\mathbf{f}$ in the above optimization problems are equivalent to the exponential stability of the closed-loop system (4).

\section{Preliminary results}

We introduce next some basic definitions and preliminary results concerning the fundamental matrix, the Cauchy formula and delay Lyapunov matrix of system (4). The fundamental matrix is defined as follows.

Definition 2 ([2]) Matrix $K(t) \in \mathbb{R}^{n \times n}$ is the fundamental matrix of system (4) if it satisfies the differential equation

$$
K^{\prime}(t)=\sum_{i=0}^{m} K\left(t-\tau_{i}\right) A_{i}(\boldsymbol{f}), t \geqslant 0
$$

and the initial conditions $K(0)=I_{n}$ and $K(t)=0$ for $t<0$.

In the next lemma, we recall the Cauchy formula of system (4), which is determined by the fundamental matrix (see, [2], or [16], p. 29).

Lemma 3 Given an initial function (5), the solution of system (4) can be expressed as

$$
x\left(t, x_{0}\right)=K(t) x_{0}, t \geqslant 0 .
$$

The following definition is used throughout the paper.

Definition 4 ([16]) System (4) satisfies the Lyapunov condition if its spectrum does not contain a root $\lambda_{0}$ such that $-\lambda_{0}$ is also a root of the spectrum.

Notice that the exponential stability of the system implies that the system satisfies the Lyapunov condition. We now introduce the parametrized delay Lyapunov matrix of system (4):

Definition 5 ([16]) Matrix $U_{W}(t, \boldsymbol{f})$ is called the parametrized delay Lyapunov matrix associated with a matrix $W(\boldsymbol{f}) \in \mathbb{R}^{n \times n}$ if it satisfies the following equalities:

(1) Dynamic property:

$$
U_{W}^{\prime}(t, \boldsymbol{f})=\sum_{i=0}^{m} U_{W}\left(t-\tau_{i}, \boldsymbol{f}\right) A_{i}(\boldsymbol{f}), t \in[0, \tau]
$$

(2) Symmetry property:

$$
\left(U_{W}(t, \boldsymbol{f})\right)^{T}=U_{W^{T}}(-t, \boldsymbol{f}), t \in[-\tau, \tau] .
$$

(3) Algebraic property:

$$
-W(\boldsymbol{f})=\sum_{j=0}^{m}\left(A_{j}^{T}(\boldsymbol{f}) U_{W}\left(\tau_{j}, \boldsymbol{f}\right)+U_{W}\left(-\tau_{j}, \boldsymbol{f}\right) A_{j}(\boldsymbol{f})\right) .
$$

Lemma 6 ([16]) If system (4) is exponentially stable, then the parametrized delay Lyapunov matrix $U_{W}(t, \boldsymbol{f})$ admits the following representation for $t \in[-\tau, \tau]$ :

$$
U_{W}(t, \boldsymbol{f})=\int_{0}^{\infty} K^{T}(\theta) W(\boldsymbol{f}) K(\theta+t) d \theta
$$

Let us introduce the vector

$$
z_{w}(t, \mathbf{f})=\left(\operatorname{vec}\left(Z_{m-1}(t, \mathbf{f})\right)^{T} \ldots \operatorname{vec}\left(Z_{-m}(t, \mathbf{f})\right)^{T}\right)^{T}
$$

where

$$
Z_{j}(t, \mathbf{f})=U_{W}(t+j h, \mathbf{f}), t \in[0, h], j=\overline{-m, m-1},
$$

and $h$ is the basic delay, i.e., without loss of generality $\tau_{k}=k h, k=\overline{1, m}$. In the next lemma, a formula for computing the delay Lyapunov matrix at points $j h, j \in$ $\{-m,-m+1, \ldots, m-2, m-1\}$ is obtained by the semianalytic method. We follow the same procedure as the one introduced in [16] for a symmetric matrix $W$. For the sake of clarity the proof is given in the appendix. 
Lemma 7 If system (4) satisfies the Lyapunov condition, then the following equality holds:

$$
z_{w}(0, \boldsymbol{f})=\left(M(\boldsymbol{f})+N(\boldsymbol{f}) e^{L(\boldsymbol{f}) h}\right)^{-1} w(\boldsymbol{f}) .
$$

Here, the matrix $L$ is the resultant matrix of the polynomials

$$
\begin{aligned}
& P_{1}(s)=I_{n}+A_{1}^{T}(\boldsymbol{f}) s+\ldots+A_{m}^{T}(\boldsymbol{f}) s^{m}, \\
& P_{2}(s)=-A_{m}^{T}(\boldsymbol{f})-A_{m-1}^{T}(\boldsymbol{f}) s-\ldots-A_{1}^{T}(\boldsymbol{f}) s^{m-1}-I_{n} s^{m}, \\
& \text { i.e., } \\
& \text { and } w^{T}(\boldsymbol{f})=\left(\begin{array}{r|r}
-\operatorname{vec}(W(\boldsymbol{f}))^{T} & 0_{1 \times(2 m-1) n^{2}}
\end{array}\right), \\
& M(\boldsymbol{f})=\left(\begin{array}{c|c}
M_{11}(\boldsymbol{f}) & M_{12}(\boldsymbol{f}) \\
\hline I_{(2 m-1) n^{2}} & 0_{(2 m-1) n^{2} \times n^{2}}
\end{array}\right), \\
& N(\boldsymbol{f})=\left(\begin{array}{c|c}
N_{11}(\boldsymbol{f}) & 0_{n^{2} \times(2 m-1) n^{2}} \\
\hline 0_{(2 m-1) n^{2} \times n^{2}} & -I_{(2 m-1) n^{2}}
\end{array}\right)
\end{aligned}
$$

where

$$
\begin{gathered}
M_{11}(\boldsymbol{f})=\left(I_{n} \otimes A_{m-1}^{T}(\boldsymbol{f}) \ldots I_{n} \otimes A_{1}^{T}(\boldsymbol{f})\right. \\
\left.I_{n} \otimes A_{0}^{T}(\boldsymbol{f})+A_{0}^{T}(\boldsymbol{f}) \otimes I_{n} A_{1}^{T}(\boldsymbol{f}) \otimes I_{n} \ldots A_{m-1}^{T}(\boldsymbol{f}) \otimes I_{n}\right),
\end{gathered}
$$

and

$$
M_{12}(\boldsymbol{f})=A_{m}^{T}(\boldsymbol{f}) \otimes I_{n}, N_{11}(\boldsymbol{f})=I_{n} \otimes A_{m}^{T}(\boldsymbol{f}) .
$$

For the computation of Lyapunov matrices for systems with non-commensurate delays the reader is referred to [15] and [8], and for large scale systems to [22].

A useful property of the delay Lyapunov matrix of system (4) is that its elements are analytic functions of the system parameters. This result is introduced in the next lemma, and the proof can be obtained by following the same argument as the one presented for proving smoothness for the one delay case in Lemma 1 in [14].

Lemma 8 If system (4) satisfies the Lyapunov condition for parameters $f=f_{0}$, then the function $\boldsymbol{f} \mapsto U_{W}(t, \boldsymbol{f})$ is analytic at $\boldsymbol{f}=\boldsymbol{f}_{0}$ for every $t \in[-\tau, \tau]$.

\section{Optimization of performance indexes}

We present in this section the main results of our contribution. First, we show that the performance indexes (3) and (8) can be expressed in terms of the delay Lyapunov matrix and that the minimax problem (7) can be reduced to the minimization of the largest eigenvalue of a symmetric matrix. Then, inspired by [14], we obtain explicit expressions of the delay Lyapunov matrix derivatives with respect to the controller gains, which are useful in the gradient computation of the objective functions. Finally, we give an explanation of the utilized numerical optimization tools.

\subsection{Performance indexes in terms of the delay Lya- punov matrix}

In the next theorem it is shown that if the closed-loop system (4) is exponentially stable, then the performance indexes (3) and (8) can be expressed in terms of a sum of delay Lyapunov matrices $U_{W}$.

Theorem 9 If the closed-loop system (4) is exponentially stable, then the performance indexes (3) and (8) can be written as

$$
J\left(\boldsymbol{f}, x_{0}\right)=x_{0}^{T} \mathcal{P}(\boldsymbol{f}) x_{0},
$$

and

$$
J_{w c}(\boldsymbol{f})=\lambda_{\max }(\mathcal{P}(\boldsymbol{f})),
$$

respectively, where $\mathcal{P}(\boldsymbol{f})$ is a symmetric matrix given by

$$
\begin{array}{r}
\mathcal{P}(\boldsymbol{f})=U_{\widehat{W}}(0, \boldsymbol{f})+\sum_{i=0}^{m-1} \sum_{j=i+1}^{m}\left(U_{W_{i j}}\left(\tau_{i}-\tau_{j}, \boldsymbol{f}\right)\right. \\
\left.+\left(U_{W_{i j}}\left(\tau_{i}-\tau_{j}, \boldsymbol{f}\right)\right)^{T}\right),
\end{array}
$$

with $\widehat{W}(\boldsymbol{f})=Q+\sum_{i=0}^{m} W_{i i}(\boldsymbol{f})$ and $W_{i j}=F_{i}^{T} C^{T} R C F_{j}$.

PROOF. Substituting the control law (2) into (3), we have

$$
\begin{aligned}
& J\left(\mathbf{f}, x_{0}\right)=\int_{0}^{\infty} x^{T}\left(t, x_{0}\right) Q x\left(t, x_{0}\right) d t \\
& +\sum_{i, j=0}^{m} \int_{0}^{\infty} x^{T}\left(t-\tau_{i}, x_{0}\right) C^{T} F_{i}^{T} R F_{j} C x\left(t-\tau_{j}, x_{0}\right) d t .
\end{aligned}
$$

By a simple change of variable and considering the initial condition in (5), the second improper integral can be written as

$I=\sum_{i, j=0}^{m} \int_{0}^{\infty} x^{T}\left(t, x_{0}\right) C^{T} F_{i}^{T} R F_{j} C x\left(t+\tau_{i}-\tau_{j}, x_{0}\right) d t$. 
Then, by using equation (9) and (13), we arrive at

$$
\begin{aligned}
J\left(\mathbf{f}, x_{0}\right) & =x_{0}^{T} \int_{0}^{\infty} K^{T}(t) Q K(t) d t x_{0} \\
+x_{0}^{T} & \sum_{i, j=0}^{m} \int_{0}^{\infty} K^{T}(t) W_{i j}(\mathbf{f}) K\left(t+\tau_{i}-\tau_{j}\right) d t x_{0} \\
& =x_{0}^{T}\left(U_{Q}(0, \mathbf{f})+\sum_{i, j=0}^{m} U_{W_{i j}}\left(\tau_{i}-\tau_{j}, \mathbf{f}\right)\right) x_{0} .
\end{aligned}
$$

From the symmetry property (11) and by rearranging the sum, we get

$$
\begin{aligned}
& U_{Q}(0, \mathbf{f})+\sum_{i, j=0}^{m} U_{W_{i j}}\left(\tau_{i}-\tau_{j}, \mathbf{f}\right)=U_{\widehat{W}}(0, \mathbf{f}) \\
+ & \sum_{i=0}^{m-1} \sum_{j=i+1}^{m}\left(U_{W_{i j}}\left(\tau_{i}-\tau_{j}, \mathbf{f}\right)+\left(U_{W_{i j}}\left(\tau_{i}-\tau_{j}, \mathbf{f}\right)\right)^{T}\right),
\end{aligned}
$$

hence,

$$
J\left(\mathbf{f}, x_{0}\right)=x_{0}^{T} \mathcal{P}(\mathbf{f}) x_{0},
$$

and equality (16) is proved. The symmetry of $\mathcal{P}(\mathbf{f})$ follows directly from the symmetry of the matrix $\widehat{W}$. Finally, equation (17) is obtained as follows:

$$
J_{w c}(\mathbf{f})=\max _{\substack{x_{0} \\\left\|x_{0}\right\| \leqslant 1}} J\left(\mathbf{f}, x_{0}\right)=\max _{\substack{x_{0} \\\left\|x_{0}\right\| \leqslant 1}} x_{0}^{T} \mathcal{P}(\mathbf{f}) x_{0}=\lambda_{\max }(\mathcal{P}(\mathbf{f})) .
$$

Remark 10 One only needs a number of $\frac{m(m+1)}{2}+1$ Lyapunov matrices in order to compute the matrix $\mathcal{P}(\boldsymbol{f})$. Furthermore, for each of them, the matrices $L(\boldsymbol{f}), M(\boldsymbol{f})$ and $N(\boldsymbol{f})$ in Lemma 7 remains the same, and the factorization of matrix $M(\boldsymbol{f})+N(\boldsymbol{f}) e^{L(\boldsymbol{f}) h}$ for computing (15) has to be done only once.

With the help of Theorem 9, the minimization problems (6) and (7) can be written as the minimization problems

$$
\min _{\mathbf{f}} x_{0}^{T} \mathcal{P}(\mathbf{f}) x_{0}
$$

when the initial condition is known, and

$$
\min _{\mathbf{f}} \lambda_{\max }(\mathcal{P}(\mathbf{f}))
$$

in the worst case of unknown initial states satisfying $\left\|x_{0}\right\| \leqslant 1$. Both problems (18) and (19) are in general non-convex, but problem (18) is smooth by Lemma 8, whereas problem (19) may be not. In fact, the largest eigenvalue of $\mathcal{P}(\mathbf{f})$ may be non-smooth at values of $\mathbf{f}$ where its multiplicity is greater than one.

A key piece in the solution of these problems is the computation of the gradient of the objective function. In the next theorem, we provide explicit expressions for computing it.

Theorem 11 If system (4) is exponentially stable, then

$$
\nabla J\left(\boldsymbol{f}, x_{0}\right)=\left(x_{0}^{T} \frac{\partial \mathcal{P}(\boldsymbol{f})}{\partial f_{1}} x_{0} \ldots x_{0}^{T} \frac{\partial \mathcal{P}(\boldsymbol{f})}{\partial f_{k}} x_{0}\right)^{T}
$$

for $\boldsymbol{f} \in \mathbb{R}^{k}$ and

$$
\nabla J_{w c}(\boldsymbol{f})=\left(v^{T}(\boldsymbol{f}) \frac{\partial \mathcal{P}(\boldsymbol{f})}{\partial f_{1}} v(\boldsymbol{f}) \ldots v^{T}(\boldsymbol{f}) \frac{\partial \mathcal{P}(\boldsymbol{f})}{\partial f_{k}} v(\boldsymbol{f})\right)^{T}
$$

for $\boldsymbol{f} \in \mathbb{R}^{k} \backslash \Omega$, where $v(\boldsymbol{f}) \in \mathbb{R}^{n}$ is the unitary eigenvector corresponding to $\lambda_{\max }(\mathcal{P}(\boldsymbol{f}))$ and $\Omega:=$ $\left\{\boldsymbol{f} \in \mathbb{R}^{k}: \mu\left(\lambda_{\max }(\mathcal{P}(\boldsymbol{f}))\right)>1\right\}$, where $\mu(\cdot)$ denotes the eigenvalue multiplicity.

PROOF. By Lemma 8, the matrix $\mathcal{P}(\mathbf{f})$ is analytic and differentiable with respect to the elements of $\mathbf{f}$, hence, equation (20) can be directly deduced from (16). We focus now on proving equation (21). As the symmetric matrix $\mathcal{P}(\mathbf{f})$ is analytic with respect to $\mathbf{f}$, we have that $\lambda_{\max }(\mathcal{P}(\mathbf{f}))$ is an analytic function of $f_{l}, l=\overline{1, k}$, at points where it is a simple eigenvalue, i.e., at $\mathbf{f} \in \mathbb{R}^{k} \backslash \Omega$, which implies the existence of the first partial derivatives of the largest eigenvalue of $\mathcal{P}(\mathbf{f})$ (see [28]). Set now $g(\mathbf{f})=$ $\lambda_{\max }(\mathcal{P}(\mathbf{f}))$, it follows from $g(\mathbf{f}) v(\mathbf{f})=\mathcal{P}(\mathbf{f}) v(\mathbf{f})$ that, for $l=\overline{1, k}$,

$\frac{\partial g(\mathbf{f})}{\partial f_{l}} v(\mathbf{f})+g(\mathbf{f}) \frac{\partial v(\mathbf{f})}{f_{l}}=\frac{\partial \mathcal{P}(\mathbf{f})}{\partial f_{l}} v(\mathbf{f})+\mathcal{P}(\mathbf{f}) \frac{\partial v(\mathbf{f})}{f_{l}}, \mathbf{f} \in \mathbb{R}^{k} \backslash \Omega$.

Premultiplying by $v^{T}(\mathbf{f})$ and taking into account that $\|v(\mathbf{f})\|=1$, we arrive at

$$
\frac{\partial g(\mathbf{f})}{\partial f_{l}}=v^{T}(\mathbf{f}) \frac{\partial \mathcal{P}(\mathbf{f})}{\partial f_{l}} v(\mathbf{f}), \mathbf{f} \in \mathbb{R}^{k} \backslash \Omega, l=\overline{1, k},
$$

and the result is immediately obtained from the gradient definition.

The partial derivatives of the matrix $\mathcal{P}(\mathbf{f})$ with respect to the optimization parameters, explicitly given by

$$
\begin{aligned}
\frac{\partial \mathcal{P}(\mathbf{f})}{\partial f_{l}}= & \frac{\partial U_{\widehat{W}}(0, \mathbf{f})}{\partial f_{l}}+\sum_{i=0}^{m-1} \sum_{j=i+1}^{m}\left(\frac{\partial U_{W_{i j}}\left(\tau_{i}-\tau_{j}, \mathbf{f}\right)}{\partial f_{l}}\right. \\
& \left.+\left(\frac{\partial U_{W_{i j}}\left(\tau_{i}-\tau_{j}, \mathbf{f}\right)}{\partial f_{l}}\right)^{T}\right), l=\overline{1, k}, \quad
\end{aligned}
$$

require the computation of the delay Lyapunov matrix derivatives with respect to the controller gains, which is addressed in the next subsection. 


\subsection{Sensitivity of the delay Lyapunov matrix}

Lemma 8 enables us to obtain the sensitivity of the delay Lyapunov matrix associated with a matrix $W(\mathbf{f})$ with respect to the elements of $\mathbf{f}$ at points $j h, j \in\{-m,-m+$ $1, \ldots, m-2, m-1\}$, from equation (15) in the next lemma.

Lemma 12 If system (4) satisfies the Lyapunov condition, then the following equality holds for $l=\overline{1, k}$ :

$$
\frac{\partial z_{w}(0, \boldsymbol{f})}{\partial f_{l}}=\left(M(\boldsymbol{f})+N(\boldsymbol{f}) e^{L(\boldsymbol{f}) h}\right)^{-1} \zeta(\boldsymbol{f})
$$

where

$$
\begin{aligned}
\zeta(\boldsymbol{f}) & =\frac{\partial w(\boldsymbol{f})}{\partial f_{l}}-\left(\frac{\partial M(\boldsymbol{f})}{\partial f_{l}}+\frac{\partial N(\boldsymbol{f})}{\partial f_{l}} e^{L(\boldsymbol{f}) h}\right) z_{w}(0, \boldsymbol{f}) \\
& -N(\boldsymbol{f}) e^{L(\boldsymbol{f}) h} \int_{0}^{h} e^{-L(\boldsymbol{f}) s} \frac{\partial L(\boldsymbol{f})}{\partial f_{l}} e^{L(\boldsymbol{f}) s} d s z_{w}(0, \boldsymbol{f}) .
\end{aligned}
$$

PROOF. We first introduce the following identity, which provides a closed-form solution for the derivative with respect to the parameters of the matrix exponential [36]:

$$
\frac{\partial e^{L(\mathbf{f}) h}}{\partial f_{l}}=e^{L(\mathbf{f}) h} \int_{0}^{h} e^{-L(\mathbf{f}) s} \frac{\partial L(\mathbf{f})}{\partial f_{l}} e^{L(\mathbf{f}) s} d s .
$$

As the Lyapunov condition holds, we have that $M+N e^{L h}$ is non singular, and from Lemma 7, we get

$$
\left(M(\mathbf{f})+N(\mathbf{f}) e^{L(\mathbf{f}) h}\right) z_{w}(0, \mathbf{f})=w(\mathbf{f})
$$

whose terms are all analytic with respect to the elements of $\mathbf{f}$ by Lemma 8 . Taking the partial derivative of the previous expression with respect to $f_{l}, l=\overline{1, k}$, on both sides, and using equation (24), we get

$$
\begin{gathered}
\left(M(\mathbf{f})+N(\mathbf{f}) e^{L(\mathbf{f}) h}\right) \frac{\partial z_{w}(0, \mathbf{f})}{\partial f_{l}} \\
=\frac{\partial w(\mathbf{f})}{\partial f_{l}}-\frac{\partial M(\mathbf{f})}{\partial f_{l}} z_{w}(0, \mathbf{f})-\frac{\partial N(\mathbf{f})}{\partial f_{l}} e^{L(\mathbf{f}) h} z_{w}(0, \mathbf{f}) \\
-N(\mathbf{f}) e^{L(\mathbf{f}) h} \int_{0}^{h} e^{-L(\mathbf{f}) s} \frac{\partial L(\mathbf{f})}{\partial f_{l}} e^{L(\mathbf{f}) s} d s z_{w}(0, \mathbf{f})=\xi(\mathbf{f})
\end{gathered}
$$

and the assertion of the lemma directly follows by inverting the matrix on the left hand side.

From the definition of the vector $z_{w}(0, \mathbf{f})$ in equation (14) and devectorization of $\frac{\partial z_{w}(0, \mathbf{f})}{\partial f_{l}}$ in equation one obtains the matrices

$$
\frac{\partial U_{W}(j h, \mathbf{f})}{\partial f_{l}}, l=\overline{1, k}
$$

where $j \in\{-m,-m+1, \ldots, m-2, m-1\}$.

Applying the above procedure for $W(\mathbf{f})=\widehat{W}(\mathbf{f})$ and $W(\mathbf{f})=W_{i j}(\mathbf{f}), i=\overline{0, m-1}, j=\overline{i+1, m}$, allows computing $\frac{\partial \mathcal{P}(\mathbf{f})}{\partial f_{l}}$ via expression $(22)$.

\subsection{Optimization algorithm}

Since the performance index $J_{w c}(\mathbf{f})$ always tends to infinity when the boundary of the feasible set (characterized by $\alpha(\mathbf{f})=0$ ) is reached, whereas the same occurs with $J\left(\mathbf{f}, x_{0}\right)$ for almost all $x_{0}$ (such that the dominant mode is present in the corresponding solution $)^{1}$, the stability constraint on $\mathbf{f}$ in the minimization problems (6) and (7) must be inactive in an optimum, and a path in the search space along which the objective function is monotonically decreasing remains in the interior of the feasible set. As a consequence, we can use unconstrained minimization techniques in order to solve Problem 1 by initializing the minimization algorithm with a stabilizing $\mathbf{f}$ and setting the objective function as

$$
g_{o b j}(\mathbf{f})= \begin{cases}\text { P.I., } & \text { if }(4) \text { is exponentially stable } \\ +\infty, & \text { otherwise }\end{cases}
$$

where P.I . is given by either (16) or (17). We notice that the stability constraint on $\mathbf{f}$ is now implicit in the objective function. Formulating the objective function in this way avoids non-stabilizing iterations in the minimization process. For instance, if the adopted algorithm generates an unstable trial step $\mathbf{f}_{u}$, then $g_{o b j}\left(\mathbf{f}_{u}\right)=+\infty$ and the line search procedure automatically reduces the step size, keeping the search inside the stability region where the algorithm was initialized.

If a stabilizing point is not known a priori, the preliminary phase of the computation of a stabilizing starting point $\mathbf{f}_{0}$ can be done via the algorithm introduced in [21], that optimizes the spectral abscissa. With the definition of $g_{o b j}$, one needs to check the stability of the system at each iteration, for which there exist different tools that can be used, see, for example, the delay Lyapunov matrix based stability criteria [7], [13], the QPmR (QuasiPolynomial Mapping Based Rootfinder) algorithm [35], spectral discretization [3], [37], or Krylov methods in the large scale setting [15].

The expressions of the gradient of the objective functions $J\left(\mathbf{f}, x_{0}\right)$ and $J_{w c}(\mathbf{f})$ in Theorem 11 enables us to

1 At the border of the stability region the Lyapunov condition does not hold, which implies that $\operatorname{det}\left(M(\mathbf{f})+N(\mathbf{f}) e^{L(\mathbf{f}) h}\right)=0$, see $[25]$. 
use gradient based algorithms in order to solve Problem 1 by minimizing $g_{o b j}$. As previously mentioned, the problem corresponding to the minimization of (16) is smooth, hence classic optimization techniques such as the BFGS (Broyden-Fletcher-Goldfarb-Shanno) quasiNewton method can be used and implemented as part of the MATLAB optimization toolbox.

The minimization of (17) is more complicated. As the largest eigenvalue of the symmetric matrix $\mathcal{P}(\mathbf{f})$ may not be smooth everywhere, standard optimization methods cannot be employed to minimize it. We use instead a variant of the BFGS algorithm targeted towards solving non-convex non-smooth problems introduced in [20]. The implementation is carried out by using the MATLAB code HANSO (Hybrid Algorithm for Nonsmooth Optimization) [20], [5]. The method only requires the evaluation of the objective function and the gradient with respect to the optimization parameters at points where it is differentiable, which is provided by Theorem 11.

Finally, since the optimization problems (18) and (19) are in general non-convex, only convergence to a local minimum can be guaranteed.

\section{$5 \quad$ Numerical examples}

Some numerical examples illustrate the presented approach for the design of controls of the form (2) that minimize the quadratic criterion (3). In each of them, we consider that the initial condition of the system is unknown, but that satisfies $\left\|x_{0}\right\| \leqslant 1$, and that the weighting matrices of the performance index (3) are given by $Q=I_{n}$ and $R=1$. The objective function $J_{w c}(\mathbf{f})$ is computed from Theorem 9 by using equation (15), and its gradient is computed from Theorem 11 by employing equation (22). The first example is taken from [33].

Example 13 Consider system (1) with matrices

$$
A=\left(\begin{array}{ccc}
-0.08 & -0.03 & 0.2 \\
0.2 & -0.04 & -0.005 \\
-0.06 & -0.2 & -0.07
\end{array}\right), B=\left(\begin{array}{c}
-0.1 \\
-0.2 \\
0.1
\end{array}\right), C=I_{3} \text {, }
$$

and a control law (2), with $F_{0}=0_{1 \times 3}, F_{1}=\left(\begin{array}{lll}f_{1} & f_{2} & f_{3}\end{array}\right)$ and $\tau=5$. The closed-loop system with the control law (2) is a time-delay system of the form (4) with matrices $A_{0}=A$ and

$$
A_{1}(\boldsymbol{f})=\left(\begin{array}{ccc}
-0.1 f_{1} & -0.1 f_{2} & -0.1 f_{3} \\
-0.2 f_{1} & -0.2 f_{2} & -0.2 f_{3} \\
0.1 f_{1} & 0.1 f_{2} & 0.1 f_{3}
\end{array}\right)
$$

By solving the minimization problem (19) with the start- ing point

$$
\boldsymbol{f}_{0}=\left(\begin{array}{lll}
0.472 & 0.505 & 0.603
\end{array}\right)^{T},
$$

that minimizes the spectral abscissa, we obtain the gain matrix

$$
\boldsymbol{f}=F_{1}^{T}=\left(\begin{array}{lll}
0.4584 & 0.3562 & 0.2454
\end{array}\right)^{T} .
$$

For this example, as only one vector gain is considered, one Lyapunov matrix associated with the matrix $\widehat{W}(\boldsymbol{f})=$ $Q+F_{1}^{T} R F_{1}$ is required in order to compute $\mathcal{P}(\boldsymbol{f})$.

Table 1 shows a comparison between the spectral abscissa and the performance index (17).

Table 1

Example 13. Trade-off between (17) and the spectral abscissa.

\begin{tabular}{|l|c|c|}
\hline & Spectral abscissa & $J_{w c}(\mathbf{f})$ \\
\hline Starting parameters $\mathbf{f}_{0}$ & -0.15 & 98.96 \\
Obtained parameters f & -0.0756 & 25.9514 \\
\hline
\end{tabular}

We consider now a Proportional-Retarded controller applied to a second order plant [34]. This class of controllers have been recently applied to have fast dynamics while minimizing noise amplification effects in [26] and [27].

Example 14 The Proportional-Retarded controller applied to a DC-servomotor in [34] can be written as system (1), with matrices

$$
A=\left(\begin{array}{cc}
0 & 1 \\
-\nu^{2} & -2 \delta \nu
\end{array}\right), B=\left(\begin{array}{l}
0 \\
b
\end{array}\right), C=\left(\begin{array}{ll}
0 & 1
\end{array}\right),
$$

where $\nu=17.6$ is the non-damped frequency, $\delta=0.0128$ is the damping factor and $b=31$ is the input gain. We consider the control law

$$
u(t)=-k_{p} x(t)+k_{r} x(t-\tau),
$$

with $k_{p}=22.57$ and $\left(\tau, k_{r}\right)$ as the design parameters. We include the delay as an optimization parameter by scaling the system. Define the time variable $\hat{t}=t / \tau$ and consider $\hat{x}(\hat{t})=x(t)$, then we obtain the closed-loop system

$$
\begin{aligned}
& \dot{\hat{x}}(\hat{t})=A_{0}(\boldsymbol{f}) \hat{x}(\hat{t})+A_{1}(\boldsymbol{f}) \hat{x}(\hat{t}-1), \\
& y(\hat{t})=C \hat{x}(\hat{t})
\end{aligned}
$$

where

$$
A_{0}(\boldsymbol{f})=f_{1}\left(\begin{array}{cc}
0 & 1 \\
-\nu^{2}-b k_{p} & -2 \delta \nu
\end{array}\right), A_{1}(\boldsymbol{f})=\left(\begin{array}{cc}
0 & 0 \\
b f_{2} & 0
\end{array}\right),
$$

and

$$
\boldsymbol{f}=\left(\begin{array}{ll}
f_{1} & f_{2}
\end{array}\right)^{T}=\left(\begin{array}{lll}
\tau & k_{r} \tau
\end{array}\right)^{T}
$$


The relation of the performance index of system (25) and (26) is given by

$$
J_{w c}(\boldsymbol{f})=f_{1} \widehat{J}_{w c}(\boldsymbol{f})
$$

where $\widehat{J}_{w c}$ is the performance index of system (26), and is computed by using expression (17) with two delay Lyapunov matrices associated with

$\widehat{W}(\boldsymbol{f})=Q+\left(k_{p}^{2}+\frac{f_{2}^{2}}{f_{1}^{2}}\right) C^{T} R C, W_{01}(\boldsymbol{f})=-k_{p} \frac{f_{2}}{f_{1}} C^{T} R C$.

The gradient of $J_{w c}(\boldsymbol{f})$ is given by

$$
\nabla J_{w c}(\boldsymbol{f})=\left(\widehat{J}_{w c}(\boldsymbol{f})+f_{1} \frac{\partial \widehat{J}_{w c}(\boldsymbol{f})}{\partial f_{1}} f_{1} \frac{\partial \widehat{J}_{w c}(\boldsymbol{f})}{\partial f_{2}}\right)^{T}
$$

The stability map of the closed-loop system in the space of parameters $\left(\tau, k_{r}\right)$ and the starting and finishing point of the optimization algorithm for two different cases are depicted in Figure 1, and the obtained results are presented in Table 2. We observe that the algorithm converges to a local optimum in the neighborhood of the parameters $\left(\tau, k_{r}\right)$ where it was initialized and remains inside the stability region.

In both cases, the decay rate of the system solution with the controller parameters obtained is slower than the decay rate with the starting parameters. It is more noticeable in the first case (green markers), where the obtained value $k_{r}=32.337$ is close to the stability boundary, which is at $k_{r}=32.5623$. This is explained by the quantity of the control energy demanded for a fast decay rate, and can be modified by either tunning the parameter $R$ of the performance index or adding an additional restriction to the location of the rightmost root.

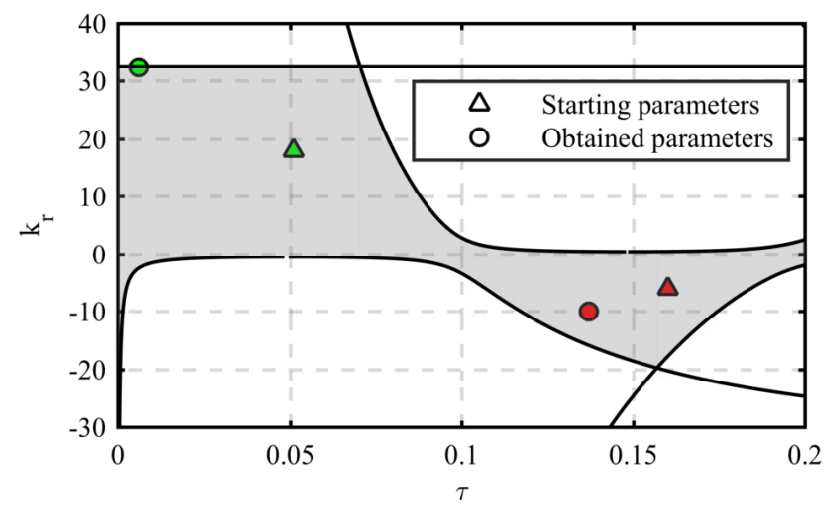

Fig. 1. Example 14. Stability map of system (26). The green and red markers correspond to the values of the first and second row of Table 2, respectively.

In [23], it is shown that a chain of $n$ integrators can always be stabilized with a controller with $n-1$ delays. In the next example, we illustrate the multiple delay case of the control law (2) by considering a triple integrator.
Table 2

Example 14. Obtained results

\begin{tabular}{|c|c|c|c|}
\hline $\mathbf{f}_{0}^{T}$ & Obtained $\mathbf{f}^{T}$ & $J_{w c}\left(\mathbf{f}_{0}\right)$ & $J_{w c}(\mathbf{f})$ \\
\hline$\left(\begin{array}{c}0.051 \\
17.96\end{array}\right)$ & $(0.00632 .337)$ & 95.226 & 15.599 \\
$(0.16-6)$ & $(0.137-9.975)$ & 255.965 & 155.273 \\
\hline
\end{tabular}

Example 15 A triple integrator admits a representation in the form of system (1) with matrices

$$
A=\left(\begin{array}{lll}
0 & 1 & 0 \\
0 & 0 & 1 \\
0 & 0 & 0
\end{array}\right), B=\left(\begin{array}{l}
0 \\
0 \\
1
\end{array}\right), C=\left(\begin{array}{lll}
1 & 0 & 0
\end{array}\right) .
$$

In [23], the following controller based on output derivatives approximation is proposed:

$$
u(t)=F_{0} y(t)+F_{1} y(t-1)+F_{2} y(t-2),
$$

where

$$
F_{0}=-3 \varepsilon-\frac{9}{2} \varepsilon^{2}-\varepsilon^{3}, F_{1}=6 \varepsilon+6 \varepsilon^{2}, F_{2}=-3 \varepsilon-\frac{3}{2} \varepsilon^{2} .
$$

The controller stabilizes the triple integrator for small values of $\varepsilon$. We address the minimization of the performance index $J_{w c}(\boldsymbol{f})$ by the control law (27) by considering both the case where $\varepsilon$ is considered as the controller parameter $(\boldsymbol{f}=\varepsilon$, Case $I)$ and the case where $F_{0}, F_{1}$ and $F_{2}$ are independent parameters $\left(\boldsymbol{f}=\left(\begin{array}{lll}F_{0} & F_{1} & F_{2}\end{array}\right)^{T}\right.$, Case II). For both cases, we set as stabilizing starting point the controller for $\varepsilon=0.08$. The results are summarized in Table 3. As expected, a better result is obtained when minimizing with respect to the three controller gains.

In order to compute $\mathcal{P}(\boldsymbol{f})$, four Lyapunov matrices are

\begin{tabular}{|c|c|c|}
\hline Case & Obtained $\mathbf{f}^{T}$ & $J_{w c}(\mathbf{f})$ \\
\hline$I$ & $\left(\begin{array}{lll}-1.5040 & 2.6144 & -1.1457\end{array}\right)$ & 66.8581 \\
\hline$I I$ & $\left(\begin{array}{lll}-1.3497 & 2.2262 & -0.9948\end{array}\right)$ & 42.7580 \\
\hline
\end{tabular}
computed associated with the matrices

$$
\begin{aligned}
\widehat{W}(\boldsymbol{f}) & =Q+\left(F_{0}^{2}+F_{1}^{2}+F_{2}^{2}\right) C^{T} R C, \\
W_{01}(\boldsymbol{f}) & =F_{0} F_{1} C^{T} R C, \\
W_{02}(\boldsymbol{f}) & =F_{0} F_{2} C^{T} R C, \\
W_{12}(\boldsymbol{f}) & =F_{1} F_{2} C^{T} R C .
\end{aligned}
$$

Table 3

Example 15. Comparison between Case I and Case II

\section{Conclusions}

A new strategy for the design of delayed output-feedback controllers that optimize a quadratic cost function is in- 
troduced. The optimization of the performance indexes (3) and (8) is reformulated as minimization problems depending on delay Lyapunov matrices. Having at hand explicit expressions of its sensitivity with respect to the controller parameters allows us to apply gradient-based techniques for the solution of the minimization problems. The effectiveness of the new approach is shown by examples of practical interest.

Some directions for future work are the integration with the recent results on the optimization of the $\mathcal{H}_{2}$ norm reported in [14] and its application to large-scale problems based on the recent methodology for the construction of delay Lyapunov matrices introduced in [22].

\section{References}

[1] I. V. Alexandrova and A. P. Zhabko. A new LKF approach to stability analysis of linear systems with uncertain delays. Automatica, 91:173-178, 2018.

[2] R. E. Bellman and K. L. Cooke. Differentialdifference equations. Academic Press, New York, 1963.

[3] D. Breda, S. Maset, and R. Vermiglio. TRACEDDE: A tool for robust analysis and characteristic equations for delay differential equations. In Niculescu SI. Sipahi R. Loiseau J.J., Michiels W., editor, Topics in Time Delay Systems: Analysis, Algorithm and Control, pages 145-155. Springer, 2009.

[4] J. V. Burke, D. Henrion, A. S. Lewis, and M. L. Overton. Stabilization via nonsmooth, nonconvex optimization. IEEE Transactions on Automatic Control, 51(11):1760-1769, 2006.

[5] J. V. Burke, A. S. Lewis, and M. L. Overton. A robust gradient sampling algorithm for nonsmooth, nonconvex optimization. Journal on Optimization, 15(3):751-779, 2005.

[6] W. H. Chen, Z.H. Guan, and X. Lu. Delaydependent output feedback guaranteed cost control for uncertain time-delay systems. Automatica, 40(7):1263-1268, 2004.

[7] A. V. Egorov. A finite necessary and sufficient stability condition for linear retarded type systems. In Proceedings of the 55th IEEE Conference on Decision and Control, pages 3155-3160, Las Vegas, USA, 2016.

[8] A. V. Egorov and V. L. Kharitonov. Approximation of delay Lyapunov matrices. International Journal of Control, 2016. DOI: 10.1080/00207179.2016.1269950.

[9] A. V. Egorov and S. Mondié. Necessary stability conditions for linear delay systems. Automatica, 50(12):3204-3208, 2014.

[10] S. H. Esfahani and I. R. Petersen. An LMI approach to output-feedback-guaranteed cost control for uncertain time-delay systems. International Journal of Robust and Nonlinear Control, 10:157-174, 2000.

[11] E. Fridman and L. Shaikhet. Stabilization by using artificial delays: An LMI approach. Automatica, 81:429-437, 2017.

[12] I. C. Gohberg and L. E. Lerer. Resultants of matrix polynomials. Bulletin of the American Mathematical Society, 82(4), 1976.

[13] M. A. Gomez, A. Egorov, and S. Mondié. Lyapunov matrix based finite stability criterion for retarded type systems. Submitted to Automatica, 2018.

[14] M. A. Gomez, A. V. Egorov, S. Mondié, and W. Michiels. Optimization of the $\mathcal{H}_{2}$ norm for single delay systems, with application to control design and model approximation. IEEE Transactions on Automatic Control, 64(2):804-811, 2019.

[15] E. Jarlebring, J. Vanbiervliet, and W. Michiels. Characterizing and computing the $\mathcal{H}_{2}$ norm of timedelay systems by solving the delay Lyapunov equation. IEEE Transactions on Automatic Control, 56(4):814-825, 2011.

[16] V. L. Kharitonov. Time-Delay Systems: Lyapunov functionals and matrices. Birkhäuser, Basel, 2013.

[17] V. L. Kharitonov, S.-I. Niculescu, J. Moreno, and W. Michiels. Static output feedback stabilization: necessary conditions for multiple delay controllers. IEEE Transactions on Automatic Control, 50(1):82-86, 2005.

[18] N. Krasovskii. On the analytic construction of an optimal control in a system with time lags. Journal of Applied Mathematics and Mechanics, 26(1):5067, 1962.

[19] W. H. Kwon and P. Park. Stabilizing and Optimizing Control for Time-Delay Systems. Springer International Publishing, 2018.

[20] A. S. Lewis and M. L. Overton. Nonsmooth optimization via quasi-newton methods. Mathematical Programming, 141:135-163, 2013.

[21] W. Michiels. Spectrum based stability analysis and stabilization of systems described by delay differential algebraic equations. IET Control Theory and Applications, 5(16):1829-1842, 2011.

[22] W. Michiels and B. Zhou. Computing delay lyapunov matrices and $\mathrm{H}_{2}$ norms for large-scale problems. arXiv:1808.08604 [math.NA], 2018.

[23] S-I. Niculescu and W. Michiels. Stabilizing a chain of integrators using multiple delays. IEEE Transactions on Automatic Control, 49(5):802-807, 2004.

[24] P. Park, Y. S. Moon, and W.H. Kwon. A stabilizing output-feedback linear quadratic control for pure input-delayed systems. International Journal of Control, 72(5):385-391, 1999.

[25] E. Plischke. Transient Effects of Linear dynamical Systems. PhD thesis, Universität Bremen, Bremen, Germany, 2005.

[26] A. Ramírez, S. Mondié, R. Garrido, and R. Sipahi. Design of proportional-integral-retarded (PIR) controllers for second-order LTI systems. IEEE Transactions on Automatic Control, 61(6):1688-1693, 2016.

[27] A. Ramírez and R. Sipahi. Multiple intentional delays can facilitate fast consensus and noise reduc- 
tion in a multiagent system. IEEE Transactions on Cybernetics, pages 1-12, 2018.

[28] F. Rellich and J. Berkowitz. Perturbation Theory of Eigenvalue Problems. Gordon and Breach, 1969.

[29] D. W. Ross and I. Flügge-Lotz. An optimal control problem for systems with differential-difference equation dynamics. SIAM Journal on Control, $7(4): 609-623,1969$.

[30] O. Santos and S. Mondié. Guaranteed cost control of linear systems with distributed delays: A complete type functionals approach. International Journal of Control, Automation, and Systems, 8(3):497-505, 2010.

[31] O. Santos, S. Mondié, and V. L. Kharitonov. Linear quadratic suboptimal control for time-delay systems. International Journal of Control, 82(1):147154, 2009.

[32] A. Selivanov and E. Fridman. An improved timedelay implementation of derivative-dependent feedback. Automatica, 98:269-276, 2018.

[33] J. Vanbiervliet, K. Berheyden, W. Michiels, and S. Vandewalle. A nonsmooth optimisation approach for the stabilisation of time-delay systems. ESAIM: Control, Optimisation and Calculus of Variations, 14:478-493, 2008.

[34] R. Villafuerte, S. Mondié, and R. Garrido. Tuning of proportional retarded controllers: theory and experiments. IEEE Transactions on Control Systems Technology, 21(3):983-990, 2013.

[35] T. Vyhlídal and P. Zítek. Mapping based algorithm for large-scale computation of quasi-polynomial zeros. IEEE Transactions on Automatic Control, 54(1):171-177, 2009.

[36] R. M. Wilcox. Exponential operators and parameter differentiation in quantum physics. Journal of Mathematical Physics, 8(4):962-982, 1967.

[37] Z. Wu and W. Michiels. Reliably computing all characteristic roots of delay differential equations in a given right half plane using a spectral method. Internal report TW 596, Department of Computer Science, KU Leuven, 2011.

\section{A $\quad$ Proof of Lemma 7}

For the sake of simplicity, we omit the argument $\mathbf{f}$ in the subsequent equations. By the dynamic property (10), for $j=\overline{0, m-1}$, we get

$Z_{j}^{\prime}(t)=\sum_{i=0}^{m} U_{W}(t+j h-i h) A_{i}=\sum_{i=0}^{m} Z_{j-i}(t) A_{i}, t \in[0, h]$.

Consider now $j=\overline{1, m}$. From the symmetry property (11), we obtain

$$
Z_{-j}(t)=U_{W}(t-j h)=\left(U_{W^{T}}(j h-t)\right)^{T}, t \in[0, h],
$$

hence

$Z_{-j}^{\prime}(t)=\left(U_{W^{T}}^{\prime}(j h-t)\right)^{T}=-\sum_{i=0}^{m} A_{i}^{T} U_{W}(-j h+t+i h)$.

Applying the definition of the matrix $Z_{j}$, we arrive at

$$
Z_{-j}^{\prime}(t)=-\sum_{i=0}^{m} A_{i}^{T} Z_{i-j}(t), t \in[0, h], j=\overline{1, m} .
$$

By using the Kronecker product property $\operatorname{vec}(E F G)=$ $\left(G^{T} \otimes E\right) \operatorname{vec}(F)$, we obtain the system of differential equations

$$
\begin{aligned}
\operatorname{vec}\left(Z_{j}^{\prime}(t)\right) & =\sum_{i=0}^{m}\left(A_{i}^{T} \otimes I_{n}\right) \operatorname{vec}\left(Z_{j-i}(t)\right), \\
\operatorname{vec}\left(Z_{-j}^{\prime}(t)\right) & =-\sum_{i=0}^{m}\left(I_{n} \otimes A_{i}^{T}\right) \operatorname{vec}\left(Z_{i-j}(t)\right),
\end{aligned}
$$

which can be written in vector form as

$$
z_{w}^{\prime}(t)=L z_{w}(t), t \in[0, h]
$$

Now, some boundary conditions of the ODE (A.1) are introduced. From the definition of the matrices $Z_{j}$ and the algebraic property (12), it follows that

$$
\operatorname{vec}\left(Z_{j}(0)\right)=\operatorname{vec}\left(Z_{j-1}(h)\right), j=\overline{-m+1, m-1},
$$

and

$$
\begin{array}{r}
\sum_{i=0}^{m-1}\left(\left(A_{i}^{T} \otimes I_{n}\right) \operatorname{vec}\left(Z_{-i}(0)\right)+\left(I_{n} \otimes A_{i}^{T}\right) \operatorname{vec}\left(Z_{i}(0)\right)\right) \\
+\left(A_{m}^{T} \otimes I_{n}\right) \operatorname{vec}\left(Z_{-m}(0)+\left(I_{n} \otimes A_{m}^{T}\right) \operatorname{vec}\left(Z_{m-1}(h)\right)\right. \\
=-\operatorname{vec}(W) . \quad(\mathrm{A} .3)
\end{array}
$$

Writing in matrix form equations (A.2) and (A.3), and taking into account that the solution of system (A.1) is $z_{w}(t)=e^{L t} z_{w}(0)$, we obtain

$$
M z_{w}(0)+N z_{w}(h)=\left(M+N e^{L h}\right) z_{w}(0)=w .
$$

Finally, as the Lyapunov condition holds $\operatorname{det}\left(M+N e^{L h}\right) \neq$ 0 ([25], p.176), and we arrive at (15). 